\title{
Spinal Cord Infarction after Cervical Transforaminal Epidural Steroid Injection: Case Report and Literature Review
}

\author{
Jangsup Moon ${ }^{a} \quad$ Hyung-Min Kwon ${ }^{b}$ \\ ${ }^{a}$ Department of Neurology, Biomedical Research Institute, Seoul National University \\ Hospital, Seoul, South Korea; ${ }^{b}$ Department of Neurology, Seoul Metropolitan \\ Government-Seoul National University Boramae Medical Center, Seoul National University \\ College of Medicine, Seoul, South Korea
}

\section{Keywords}

Transforaminal epidural steroid injection · Spinal cord infarction · Complication

\begin{abstract}
Introduction: Transforaminal epidural steroid injection (TFESI) is a widely used nonsurgical procedure in the treatment of patients with radiculopathy. It is efficacious in relieving pain, but a number of complications are being reported. Recently, increasing frequency of major complications, such as spinal cord infarction and cerebral infarction, has been reported with the use of a particulate steroid within fluoroscopic-guided procedures. Methods: We report a 49-year-old man with a history of chronic cervical radiculopathy, who experienced a devastating complication after TFESI. Results: After 2 min of regular TFESI, the patient abruptly experienced muscle weakness in both upper extremities and within 5 min the patient became quadriplegic. Despite active rehabilitation, the patient remained bed-ridden 4 years after the catastrophic event. To our knowledge, this is the first reported case of spinal cord infarction that occurred after TFESI in Korea. Conclusion: Considering the risk of dreadful complications, which appear in an unpredictable manner, TFESI with fluoroscopic guidance should be done only with a nonparticulate steroid.




\section{Introduction}

Transforaminal epidural steroid injection (TFESI) is widely performed as a nonsurgical management of patients with radicular pain syndromes. This procedure allows direct delivery of injected corticosteroids to the nerve root and enables suppression of the surrounding inflammatory response, which is responsible for the radicular pain $[1,2]$. While there are few complications after TFESI, an increasing number of devastating complications such as spinal cord infarction, cerebral infarction and death are being reported with the use of a particulate steroid within fluoroscopic-guided procedures [3, 4]. Here, we report the first case of a patient who experienced spinal cord infarction after cervical TFEFI in Korea.

\section{Case Report}

A 49-year-old man was transferred to our neurology department after abrupt onset of quadriparesis after a cervical TFESI. He was relatively healthy except for a long history of cervical radiculopathy. He visited the pain clinic of our hospital due to the persistent radiating pain and received a left-sided cervical TFESI at the C6-C7 level. The procedure was performed by an expert anesthesiologist. Fluoroscopy was utilized to guide the injection needle into the foramina. After confirmation of contrast around the nerve root and epidural space and the absence of vascular uptake, $20 \mathrm{mg}$ of triamcinolone and $2 \mathrm{~mL}$ of $0.125 \%$ levobupivacaine were injected. Approximately 2 min after the procedure, the patient complained of weakness of both upper extremities. After $5 \mathrm{~min}$, he described weakness of both lower extremities. On the initial neurological examination, he was quadriplegic with motor power less than grade I in all 4 extremities. His sensory function was absent below the C2 level. Cranial nerve examination was normal and respiration was intact. The initial diffusion magnetic resonance imaging (MRI) revealed an extensive ischemic lesion in the spinal cord, below $\mathrm{C} 2$ to the upper thoracic level. Follow-up MRI taken 3 days after the event demonstrated more significant signal changes in the diffusion-weighted images and T2-weighted images (Fig. 1). The brain MRI was normal. Although the patient's motor power continued to improve with active rehabilitation, he remained bed-ridden 4 years after the catastrophic event.

\section{Discussion}

TFESI is widely performed in patients with cervical and lumbosacral radiculopathy. TFESI reduces pain significantly - although the effect is transient - and decreases the need for surgery [3]. The reported incidence of periprocedural complications of TFESI is low. Complications include dural puncture, trauma to the spinal nerve, infection, and allergic reaction to the medication [3]. However, scrutiny of the literature reveals the existence of more severe procedure-related complications, which can leave permanent sequelae. To date, more than 15 cases of spinal cord infarction and 30 cases of cerebral infarction have been reported after TFESI. Fifteen deaths associated with this procedure have been reported [4]. However, the accurate rate of the major complication is unknown, because a lot of cases may not have been reported $[3,4]$.

The suggested mechanism of the ischemic complications of the central nervous system is that inadvertent intra-arterial injection of a particulate corticosteroid creates an embolus 


\section{Case Reports in Neurology}

Moon and Kwon: Spinal Cord Infarction after Cervical Transforaminal Epidural Steroid Injection: Case Report and Literature Review

and subsequently leads to ischemic lesions in the anterior spinal or vertebral artery territories $[3,5]$. A few prospective studies and case reports support the embolic mechanism via the intra-arterial injection of a particulate steroid [6,7]. Although intravascular injections most likely result from incorrect needle placement, a number of studies have demonstrated that the intra-arterial injection of a steroid into a radicular artery is possible, even with proper needle placement [7]. The type of needle used for the procedure can also influence the rate of the intravascular injection [8]. Moreover, variable anastomoses exist between vertebral and cervical arteries, so that steroid particles could enter into the vertebral circulation via cervical arteries and cause cerebellar and brainstem infarcts [9]. Two reports have described patients who had a previous history of spinal surgery and experienced spinal cord infarction after TFESI $[9,10]$. Previous surgery may have given rise to more anastomoses around the spinal canal in these patients. The corticosteroid seems to play a main role in the ischemic complications, with the steroid subtype being influential. Particulate steroids, such as methylprednisolone, triamcinolone, and betamethasone, have been reported to cause ischemic complications, while the nonparticulate steroid, dexamethasone, has not. The large particle size of some steroids may drive coalescence into larger aggregates that could induce embolic infarcts [3].

Other proposed mechanisms are vertebral artery dissection resulting from a direct needle injury or malposition of the needle to intramedullar spinal cord [11]. Also, accidental laceration of an intervertebral disc by the needle may lead to fibrocartilaginous embolus [12] and accidental intraosseous injection of the steroid due to osteopenia may cause intravascular penetration of the steroid [5]. However, our patient showed normal appearance of the vertebral artery.

To date, no major ischemic complication has been reported after TFESI in Korea. One patient had experienced cervical subdural hematoma after cervical TFESI, which led to transient quadriplegia [13]. Another patient had experienced motor weakness in both lower extremities 2 days after cervical TFESI [14]. Spinal cord diffusion MRI was not performed, but considering the delayed onset of symptoms, spinal cord infarction was not suspected.

Our patient encountered a catastrophic complication although the procedure was performed in the same manner as routine procedures. We suppose that a particulate steroid, triamcinolone, inadvertently entered the arterial system of the spinal cord and subsequently induced massive embolic infarction of the spinal cord. In 2015, a multidisciplinary working group announced a safeguard recommendation in effort to improve the safety of epidural steroid injections [11]. According to the recommendation, all cervical procedures should be performed using image guidance, and contrast medium should be injected under real-time fluoroscopy before injecting any substance. Particulate steroids should not be used in cervical TFESI. Computed tomography (CT) guidance offers several advantages over fluoroscopic guidance [15]. CT guidance can avoid cannulation of small arteries such as vertebral artery and also radical-medullar and other muscular cervical arteries that can sometimes give anatomic branches to radical-medullar arteries. Intramedullar needle placement can be avoided by CT guidance but not by fluoroscopic guidance alone.

Considering the risk of dreadful complications with a particulate steroid, and in this case triamcinolone, TFESI with fluoroscopic guidance should be done only with a nonparticulate steroid, like dexamethasone. CT guidance of the procedure can further decrease the chance of major complications. 
Moon and Kwon: Spinal Cord Infarction after Cervical Transforaminal Epidural Steroid Injection: Case Report and Literature Review

\section{Statement of Ethics}

The authors have no ethical conflicts to disclose.

\section{Disclosure Statement}

There are no conflicts of interest to disclose.

\section{References}

1 Boswell MV, Hansen HC, Trescot AM, Hirsch JA: Epidural steroids in the management of chronic spinal pain and radiculopathy. Pain Physician 2003;6:319-334.

-2 Park SY, An HS, Moon SH, Lee HM, Suh SW, Chen D, et al: Neuropathic pain components in patients with lumbar spinal stenosis. Yonsei Med J 2015;56:1044-1050.

-3 Scanlon GC, Moeller-Bertram T, Romanowsky SM, Wallace MS: Cervical transforaminal epidural steroid injections: more dangerous than we think? Spine 2007;32:1249.

4 Benny B, Azari P, Briones D: Complications of cervical transforaminal epidural steroid injections. Am J Phys Med Rehabil 2010;89:601.

-5 Wybier M: Transforaminal epidural corticosteroid injections and spinal cord infarction. Joint Bone Spine 2008;75:523.

-6 Baker R, Dreyfuss P, Mercer S, Bogduk N: Cervical transforaminal injection of corticosteroids into a radicular artery: a possible mechanism for spinal cord injury. Pain 2003;103:211-215.

7 Verrills P, Nowesenitz G, Barnard A: Penetration of a cervical radicular artery during a transforaminal epidural injection. Pain Med 2010;11:229-231.

-8 Hong J, Jung S, Chang H: Whitacre needle reduces the incidence of intravascular uptake in lumbar transforaminal epidural steroid injections. Pain Physician 2015;18:325-331.

-9 Huntoon MA, Martin DP: Paralysis after transforaminal epidural injection and previous spinal surgery. Reg Anesth Pain Med 2004;29:494-495.

$\$ 10$ Houten JK, Errico TJ: Paraplegia after lumbosacral nerve root block: report of three cases. Spine J 2002;2:70-75.

11 Rathmell JP, Benzon HT, Dreyfuss P, Huntoon M, Wallace M, Baker R, et al: Safeguards to prevent neurologic complications after epidural steroid injections: consensus opinions from a multidisciplinary working group and national organizations. Anesthesiology 2015;122:974-984.

$\$ 12$ Meyer HJ, Monticelli F, Kiesslich J: Fatal embolism of the anterior spinal artery after local cervical analgetic infiltration. Forensic Sci Int 2005;149:115-119.

13 Lee JK, Chae KW, Ju CI, Kim BW: Acute cervical subdural hematoma with quadriparesis after cervical transforaminal epidural block. J Korean Neurosurg Soc 2015;58:483-486.

14 Han M: Low extremity weakness after cervical epidural steroid injection in previous spinal surgery patient: a case report. Korean J Anesthesiol 2008;54:593-597.

15 Wald JT, Maus TP, Diehn FE, Kaufmann TJ, Morris JM, Murthy NS, et al: CT-guided cervical transforaminal epidural steroid injections: technical insights. J Neuroradiol 2014;41:211-215 


\section{Case Reports in Neurology}

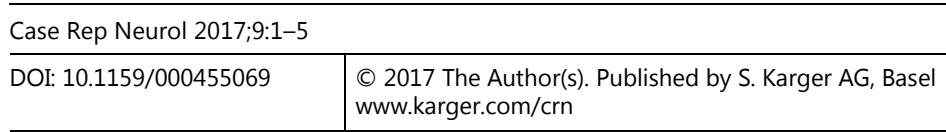

Moon and Kwon: Spinal Cord Infarction after Cervical Transforaminal Epidural Steroid Injection: Case Report and Literature Review
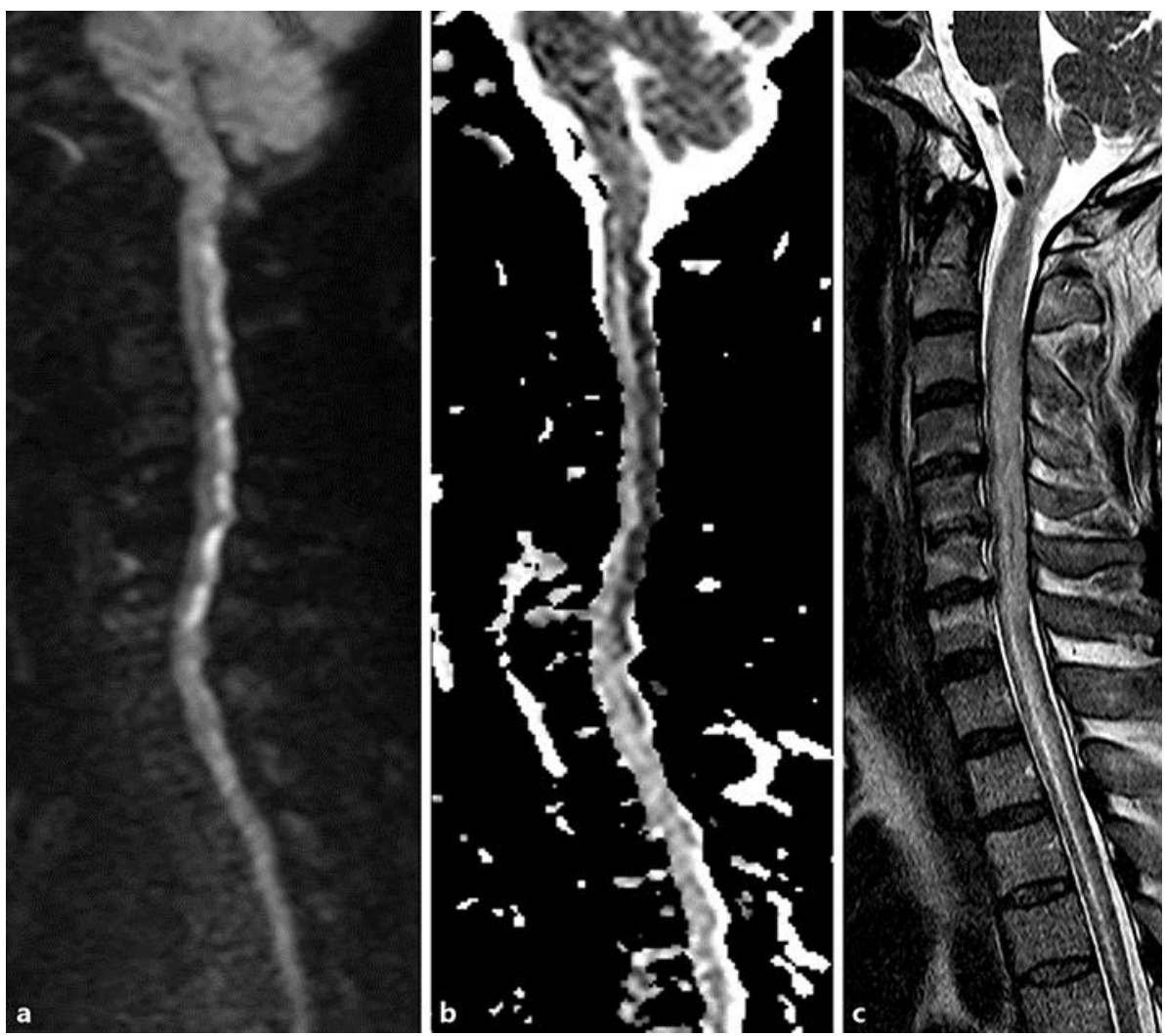

Fig. 1. Spine MRI data of the patient. A diffusion-weighted image (a) and an apparent diffusion coefficient image (b) show an extensive ischemic infarction in the spinal cord, below C2 to the upper thoracic level. A T2-weighted image (c) demonstrates high signal changes and swelling of the spinal cord. 